\title{
Long uvula: an unusual cause of chronic cough
}

Chronic cough is a distressing symptom for patients and parents in paediatric practice. Investigations of the child with persistent cough can be simple or complex depending on the underlying cause. There are some unusual reasons for chronic cough, which need to be kept in mind when investigating the cause. A case of a long, floppy uvula causing chronic cough through mechanical irritation of upper airway is reported.

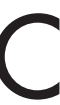
ough is an important protective reflex of the body. But when this protective reflex turns into persistent cough, a common problem in paediatric practice, it can become an extremely distressing symptom for patients and parents. Infection is one of the most common causes for acute cough, whereas chronic inflammation and mechanical irritation are common causes of chronic cough. In paediatric practice, asthma is a prime example of persistent cough.

There are some unusual causes of chronic cough, which if not looked for can lead to unnecessary investigations and treatment. We would like to present an unusual cause of chronic cough to illustrate our point.

\section{CASE REPORT}

A 1 year old boy was referred with the history of spasmodic cough since the age of 6 weeks. This distressing cough usually ended in vomiting with variable symptomatic relief. The coughing episodes were mainly observed during the daytime and often precipitated by a semisolid or solid diet. There was no history of cold, temperature, or breathlessness associated with it.

Apart from this distressing symptom, he was asymptomatic and thriving well. His growth was appropriate for his age. He was born by emergency lower segment caesarean section with birth weight of $3200 \mathrm{~g}$. His neonatal period was uneventful. He was fully immunised and had not come in contact with whooping cough.

The patient's father is from Morocco and as a young child he underwent uvulectomy. This is a common ritual performed for all babies in Morocco. There was no other relevant family history.

Apart from a long uvula (fig 1), his clinical examination was completely normal.

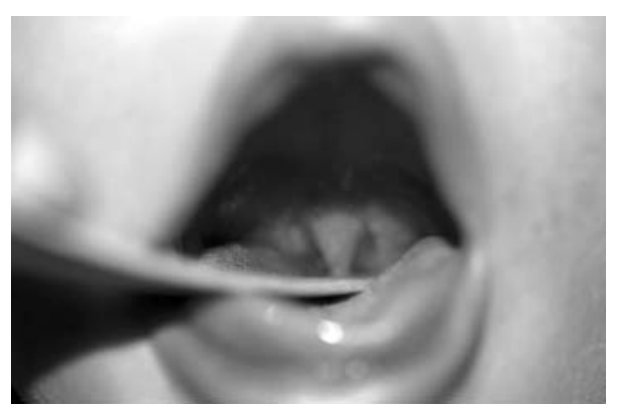

Figure 1 Patient's mouth showing long uvula.

\section{Investigations}

With the initial differential diagnosis of whooping cough or gastro-oesophageal reflux, a series of investigations were carried out. A full blood count, blood culture, nasal swab, chest radiograph, and oesophageal pH study were performed and were reported as normal.

With a view to ruling out any anatomical problem or pharyngeal incoordination, a barium study with video fluoroscopy was performed. This was again reported as normal but interestingly, the radiologist commented on his elongated uvula, which was seen prominently on the film.

\section{Progress}

He continues to have coughing bouts that end in vomiting. In spite of this, he is thriving well with a good appetite.

At present he is awaiting an ear, nose, and throat appointment for an opinion and possible uvulectomy.

\section{DISCUSSION}

The uvula is an appendage of soft palate and is formed by the fusion of the two halves of the soft palate. It is an important structure in producing a tight seal in the pharynx during speech and feeding. An elongated uvula can flop down and touch various structures in the upper airway including the posterior pharyngeal wall, epiglottis, and vocal cords. Irritation of these structures can lead to chronic cough.

There are case reports of the uvula causing apnoea due to irritation of the epiglottis or vocal cords. Uvulectomy led to relief from these symptoms. ${ }^{12}$ In Africa, especially in the Muslim community, uvulectomy is an ancient ritual/tradition and is as common as circumcision. There are various case reports and commentaries from the literature in 1970-80. Uvulectomies are normally performed by traditional healers with indifferent results..$^{3-5}$

This case report illustrates the importance of eliciting a family history in reaching the diagnosis as well as the need for thorough examination for unusual signs, which can point towards the aetiology. Unusual aetiology must be kept in mind if the usual causes do not seem to solve the problem.

\section{Authors' affiliations}

V Pai, Royal Alexandra Children's Hospital, Dyke Road, Brighton BN1 3JN, UK

H Thomas, C Stewart, Southmead Hospital, Westbury-on-Trym, Bristol, UK

Correspondence to: Dr Pai, binapai@hotmail.com

Submitted 12 June 2003

Accepted 26 June 2003

\section{REFERENCES}

1 Najada A, Weinberg M. Unusual cause of chronic cough in a 4 year old child. Pediatr Pulmonol 2002;34:144-6.

2 Miller FR, Tucker HM. An elongated uvula producing chronic cough. Otolaryngol Head Neck Surg 1993;109:954-5.

3 Manni JJ. Uvulectomy, traditional surgical procedure in Tanzania. Ann Trop Med Parasitol 1984;78:49-53.

4 Einterez EM, Einterezz RM. Traditional uvulectomy in northern Cameroon. Lancet 1994;343:1644.

5 Wind J. Cross cultural and anthropological reflection on African uvulectomy. Lancet 1984;ii:1267-8. 